\title{
SIMULACION DE FLUJO 1D EN CANALES ABIERTOS
}

\author{
Víctor A. Yzocupe \\ Laboratorio de Fluidodinámica Computacional, Departamento de Ingeniería Mecánica de Fluidos, \\ Facultad de Ciencias Físicas, Universidad Nacional Mayor de San Marcos. Lima, Perú
}

\section{Resumen}

Se presenta la formulación de un modelo numérico unidimensional para la simulación de flujo en ríos y canales. El modelo es general y flexible; porque permite simular un amplio rango de condiciones de flujo, tales como flujo uniforme y no uniforme, estacionario y no estacionario. El modelo no considera problemas de filtración, transporte de sedimentos, lluvia, evaporación, ni viento.

El modelo se basa en la aproximación numérica de las ecuaciones gobernantes del flujo en canales abiertos (ecuaciones de Saint Venant) mediante el esquema de diferencias finitas implícito de Preissmann. Las variables de cálculo son el caudal (Q) y el nivel de la superficie líquida (Z). Los algoritmos aplicados en los segmentos del tramo en estudio mas las condiciones de frontera componen un sistema de ecuaciones lineales, el mismo que se resuelve mediante el método de Doble Barrido.

Se presentan algunas pruebas esquemáticas para un canal rectangular prismático. Se han realizado pruebas para flujo estacionario uniforme y no uniforme, además de pruebas para flujo no estacionario, las mismas que comprueban la bondad del modelo presentado.

Palabras claves: Modelo numérico, hidráulica de canales, hidráulica computacional, flujo unidimensional.

\begin{abstract}
It presents the formulation of a one-dimensional numerical model for simulation of flow in rivers and channels. The model is general and flexible; because it permits to simulate a broad range of flow conditions, such as uniform and non-uniform flow, steady and unsteady. The model does not consider problems of filtration, sediment transport, rainfall, evaporation, neither wind.

The model is based in the numerical approximation of the governing equations of flow in open channels (Saint Venant's equations) using the implicit finite difference Preissmann scheme. The computation variables are the discharge $(\mathrm{Q})$ and the water surface level $(\mathrm{Z})$. The algorithms applied in the segments of the reach under study plus the boundary conditions compose one system of lineal equations, which is solved using the Double Sweep method.
\end{abstract}

It presents some schematic tests for a prismatic rectangular channel. There are some tests for steady uniform and non-uniform flow, also some tests for non-steady flow, which demonstrate the goodness of the model.

Keywords: Numerical model, hydraulics of channels, computational hydraulics, one-dimensional flow.

\section{Introducción}

El modelamiento de flujo unidimensional en ríos y canales es una potente herramienta para proyectos de investigación o de ingeniería en los que se necesita conocer la distribución de caudales y niveles a lo largo de un sistema fluvial o de canales de riego.
En hidráulica fluvial, existen muchos casos donde los parámetros del flujo varían con el tiempo. Un caso de estos, es el desplazamiento de ondas de crecida en ríos, una onda de crecida es el aumento lento y gradual del nivel de la superficie en un tramo de canal. Otro caso, es el desplazamiento de la onda generada por el rompimiento de una presa [8]. 
Si tenemos un tramo de canal regular con muy baja resistencia y una onda de crecida moviéndose a través de éste; entonces, la configuración de la onda permanecerá casi inalterada. Sin embargo, en un cauce real, la resistencia es alta debido a su irregularidad; por tanto, la configuración de la onda se modifica continuamente a lo largo de su recorrido. La determinación de esta modificación se conoce como desplazamiento de crecidas y se puede aplicar tanto a canales como a embalses [4].

El desplazamiento de crecidas es útil para la implementación de un programa de control de crecidas y para la operación de un servicio de predicción de inundaciones. El desplazamiento de crecidas se conoce como un procedimiento que simula el proceso y produce un registro continuo en varias secciones de control elegidas sobre el curso de agua, teniendo como punto de partida un hidrograma de crecidas en la frontera aguas arriba.

Para la solución del desplazamiento de crecidas existen dos técnicas de solución, los métodos hidrológicos y los métodos hidráulicos. Los métodos hidrológicos se basan en una simple relación descarga-almacenamiento y el principio de continuidad; en cambio, los métodos hidráulicos se basan en las ecuaciones diferenciales de conservación de masa y de conservación de la cantidad de movimiento [8].

Los términos "canal", "tramo" o "ramal" se usan indistintamente para definir una cierta longitud de canal, con características geométricas o hidráulicas homogéneas o que esta controlado por alguna condición de frontera externa o interna. La subdivisión de un canal o tramo se conoce como un "segmento". Una "red" es un sistema compuesto por varios canales. El modelo presentado permite simular el flujo en canales singulares o canales en serie.

- Canal simple o singular:

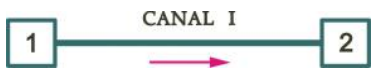

1 NODO EXTERNO

- Canales en serie:

(2) NODO INTERNO

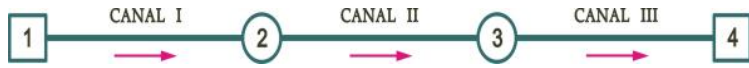

\section{Formulación del Modelo Numérico}

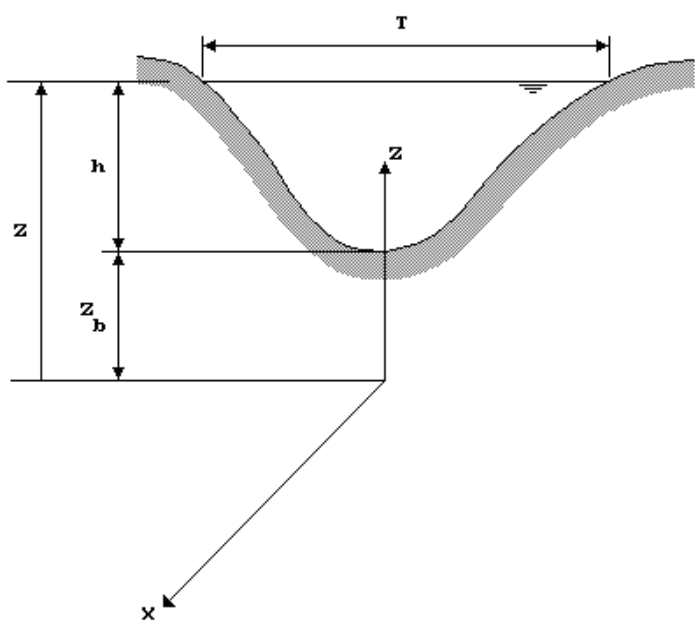

Fig. 1. Sistema de Coordenadas

Las ecuaciones que describen el flujo no estacionario unidimensional en canales abiertos son la ecuación de continuidad y la ecuación de movimiento. El desarrollo de la ecuación de continuidad está basado en el principio de conservación de la masa, mientras que la ecuación de movimiento está basada directamente en la segunda Ley de Newton, en lugar de utilizar la conservación de energía o de momentum [6].

El desarrollo de las ecuaciones gobernantes del flujo en canales, se sustenta en un sistema de coordenadas $X-Z$, cuyo origen vertical se puede ubicar en el nivel de la frontera aguas abajo o en forma más general en el nivel medio del mar.

El eje $X$ se utiliza para representar la distancia longitudinal del canal y es positivo hacia aguas abajo del canal. El eje $Z$ representa el nivel de la superficie líquida, y $Z_{b}$ es el nivel del fondo del canal, $h$ representa la altura neta de la columna de agua.

\subsection{Ecuaciones Gobernantes}

Estas ecuaciones diferenciales parciales de continuidad y de movimiento, también se conocen como las Ecuaciones de Aguas Rasas o de Saint Venant. La descarga $(Q)$ y el nivel de la superficie del agua ( $Z$ ) son las variables dependientes.

Ecuación de Conservación de Masa [6]: 


$$
T \frac{\partial Z}{\partial t}+\frac{\partial Q}{\partial x}=0
$$

Ecuación de Conservación de Cantidad de Movimiento [6]:

$$
\frac{\partial Q}{\partial t}+\frac{\partial}{\partial x}\left(\beta \frac{Q^{2}}{A}\right)+g A \frac{\partial Z}{\partial x}+g A S_{f}=0
$$

donde $\beta$ y $S_{\mathrm{f}}$ se definen como [3]:

$$
\begin{gathered}
\beta=\frac{1}{U^{2} A} \int_{0}^{T} u^{2} d A ; \quad \beta=\beta(Z) \\
S_{f}=\frac{n^{2} Q^{2}}{A^{2} R^{4 / 3}}
\end{gathered}
$$

y el término no lineal de la ecuación (2) se descompone así [3]:

$$
\begin{gathered}
\frac{\partial}{\partial x}\left(\beta \frac{Q^{2}}{A}\right)=2 \beta \frac{Q}{A} \frac{\partial Q}{\partial x}-\beta \frac{Q^{2}}{A^{2}} \frac{\partial A}{\partial x} \\
\frac{\partial A}{\partial x}=\frac{\partial A}{\partial Z} \frac{\partial Z}{\partial x}+\left.\frac{\partial A}{\partial x}\right|_{Z=c t e}=T \frac{\partial Z}{\partial x}+\left.\frac{\partial A}{\partial x}\right|_{Z=c t e} \\
T=T(Z), \quad A=A(Z)
\end{gathered}
$$

Reemplazando todas las anteriores ecuaciones en (2) obtenemos:

$$
\begin{aligned}
& \frac{\partial Q}{\partial t}+2 \beta \frac{Q}{A} \frac{\partial Q}{\partial x}+\left(g A-\beta \frac{Q^{2}}{A^{2}} T\right) \frac{\partial Z}{\partial x} \\
& -\left.\beta \frac{Q^{2}}{A^{2}} \frac{\partial A}{\partial x}\right|_{Z=c t e}+g \frac{n^{2}|Q| Q}{A R^{4 / 3}}=0
\end{aligned}
$$

En estas ecuaciones, la distancia longitudinal a lo largo del thalweg del canal $(x)$, y el tiempo transcurrido $(t)$, son las variables independientes (la distancia longitudinal $(x)$, y el caudal o descarga $(Q)$, son positivas en la dirección aguas abajo). Las otras cantidades se definen como:

$Z$ nivel de la superficie líquida, referida a un datum $=Z_{b}+h$,

$Z_{\mathrm{b}}$ nivel del lecho, referida a un datum,

$h$ profundidad vertical del flujo o altura de lámina de agua,

$S_{\mathrm{f}}$ pendiente de fricción,

$\beta$ coeficiente de distribución de velocidad o de Boussinesq,

$g$ aceleración gravitacional,
A área mojada de la sección transversal,

$R$ radio hidráulico de la sección transversal,

$P$ perímetro mojado de la sección transversal,

$T$ ancho total de la superficie líquida, transversal a la dirección del flujo,

$u$ velocidad del flujo en un punto de la sección transversal,

$U$ velocidad media del flujo $=Q / A$,

$n$ coeficiente de rugosidad de Manning.

El radio hidráulico $(R)$ se utiliza en la ecuación (3) y en todas las demás ecuaciones; pero, cuando el canal tiene una configuración geométrica arbitraria (canal natural), se puede sustituir el radio hidráulico por la profundidad hidráulica. Esta aproximación $(R \approx A / T)$ se asume válida para cuerpos de agua poco profundos, y se utiliza por la facilidad de calcular el ancho de la superficie líquida en lugar del perímetro hidráulico.

El coeficiente de Boussinesq, $\beta$, también llamado el coeficiente de momentum, está presente en la ecuación de movimiento para tomar en cuenta las distribuciones de velocidad no uniformes en las secciones transversales.

Las ecuaciones (1) y (5) describen, en general, el flujo no permanente en un canal de sección arbitraria teniendo áreas de transporte y de almacenamiento (o solamente de transporte). En su formulación, se asume que el agua es de densidad homogénea, que la presión hidrostática prevalece en todo el canal, que la pendiente de fondo del canal es pequeña y uniforme, que no hay procesos de transporte de sedimentos en el lecho del canal (no ocurre erosión ni sedimentación), que la geometría del tramo es suficientemente uniforme para permitir la aproximación unidimensional, y que la resistencia por fricción es la misma como en el flujo permanente, permitiendo el uso de la ecuación de Chézy o Manning.

\subsection{Esquema de Diferencias Finitas Preissmann}

Existen numerosos métodos numéricos para producir soluciones aproximadas de las ecuaciones de flujo. En este trabajo, las ecuaciones de flujo serán discretizadas mediante el esquema de diferencias finitas implícito de Preissmann. Esta técnica, permite que el modelo 
utilice segmentos de diferentes longitudes y un esquema que va desde centrado hasta totalmente adelantado en el tiempo.

El método de solución implícito se emplea debido a su eficiencia inherente y propiedades de estabilidad superior. Es posible agregar un procedimiento de iteración opcional controlable por el usuario para mejorar la exactitud de los resultados.

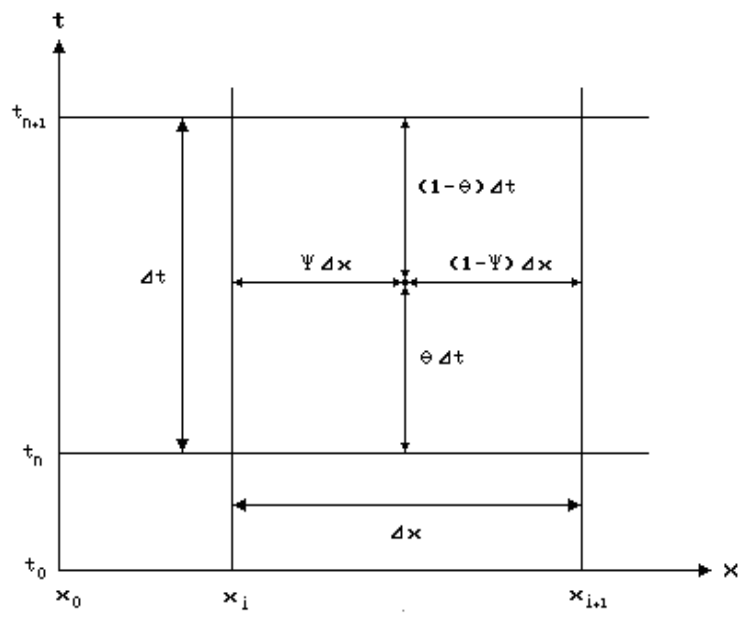

Fig. 2. Grilla espacio-temporal Esquema Preissmann

El sistema de grilla espacio-temporal de la figura 2 muestra la región en que las ecuaciones de flujo son resueltas. Las derivadas temporal y espacial del valor funcional, $f$, que representa la variable dependiente, nivel (elevación de la superficie líquida) o caudal, son discretizadas de la siguiente manera [2]:

$$
\begin{gathered}
\frac{\partial f}{\partial t} \approx(1-\psi) \frac{f_{i}^{n+1}-f_{i}^{n}}{\Delta t}+\psi \frac{f_{i+1}^{n+1}+-f_{i+1}^{n}}{\Delta t} \\
\Delta t=\Delta t_{1}=\ldots=\Delta t_{n} \\
\frac{\partial f}{\partial x} \approx(1-\theta) \frac{f_{i+1}^{n}-f_{i}^{n}}{\Delta x_{i}}+\theta \frac{f_{i+1}^{n+1}-f_{i}^{n+1}}{\Delta x_{i}}
\end{gathered}
$$

Donde, $0 \leq \psi \leq 1$, y $0.5 \leq \theta \leq 1$ son factores de ponderación utilizados para especificar la posición temporal y espacial, respectivamente, dentro del incremento de tiempo $\Delta \mathrm{t}_{\mathrm{n}}$ e incremento de distancia $\Delta \mathrm{x}_{\mathrm{i}}$ en el cual la derivada $\mathrm{y}$ las funciones serán evaluadas. Tomando $\psi=\frac{1}{2}$, produce una derivada temporal en la posición espacial $i+\frac{1}{2}$. Similarmente, cuando $\theta=\frac{1}{2}$ la derivada espacial esta centrada en la dirección temporal $n+\frac{1}{2}$. Los errores de truncamiento son del orden $\Delta t^{2}$ y $\Delta x^{2}$; pero tomando $\theta>\frac{1}{2}$ se introducen errores de truncamiento que producen disipación numérica. Las derivadas temporales normalmente son calculadas con $\psi=\frac{1}{2}$, aunque otros valores pueden ser ventajosos cuando se utilizan segmentos de longitudes desiguales [2].

De una manera similar al tratamiento de la derivada espacial, el área de la sección transversal, el ancho de la superficie libre, el radio hidráulico, y las descargas en forma no derivativa, denotadas por $f(x, t)$, se discretizan como sigue [5]:

$$
f(x, t) \approx(1-\chi) \frac{f_{i+1}^{n}+f_{i}^{n}}{2}+\chi \frac{f_{i+1}^{n+1}+f_{i}^{n+1}}{2}
$$

El factor de ponderación $\chi$ puede ser asignado en el rango $0 \leq \chi \leq 1$. Así, estos valores funcionales pueden ser representados en cualquier nivel de tiempo como las derivadas espaciales.

La determinación de valores apropiados para estos parámetros es importante porque ellos tienen efecto en la precisión, convergencia, y estabilidad del modelo. Tales valores son la determinación del incremento de tiempo $(\Delta \mathrm{t})$, la longitud de los segmentos del canal $(\Delta x)$, y la selección de los apropiados factores de ponderación del esquema Preissmann.

La solución numérica de las ecuaciones de flujo en un sistema de grilla rectangular $\mathrm{x}$-t, impone una restricción en la determinación del incremento de tiempo basado en la longitud de los segmentos. Esta restricción es aplicable, en un sentido matemático riguroso, a la técnica de diferencias finitas explícita y se denomina Condición de Courant-Friedrichs-Lewy [2],

$$
\Delta t \leq \frac{\Delta x}{|U \pm \sqrt{g h}|}
$$

En esta relación, $\Delta t$ es el incremento de tiempo, $\Delta x$ es la longitud del segmento, $U$ es la velocidad media del flujo, $g$ es la aceleración de la gravedad y $h$ es la profundidad del flujo. Esta 
condición asegura condiciones de cálculo estable y requiere excesiva cantidad de tiempo de cómputo; por esta razón las técnicas de solución implícitas ofrecen ventajas económicas. No obstante, la Condición de Courant, la cual es una función de la celeridad de onda $(c=\sqrt{g h})$ y la velocidad del flujo, constituyen un valioso índice cuando seleccionamos el incremento de tiempo para una solución implícita. Sin embargo, incrementar $\Delta \mathrm{t}$ puede también degradar la precisión de la simulación y por tanto, rendir resultados inútiles.

\subsection{Discretización de las Ecuaciones Gobernantes}

Las ecuaciones diferenciales parciales de flujo (1) y (5) son transformadas en expresiones discretas mediante la aplicación del esquema de diferencias finitas implícito de Preissmann, utilizando los operadores definidos en las ecuaciones (6), (7) y (8). Se utiliza la tilde ( ) para denotar las cantidades tomadas como constantes locales, las que serán actualizadas a través de las iteraciones en el proceso de cálculo.

La ecuación de continuidad se reduce a:

$$
a Z_{i}^{n+1}-Q_{i}^{n+1}+a Z_{i+1}^{n+1}+Q_{i+1}^{n+1}=b
$$

donde,

$$
\begin{aligned}
& a=\frac{\tilde{T} \Delta x_{i}}{2 \theta \Delta t} \\
& b=a\left(Z_{i}^{n}+Z_{i+1}^{n}\right)+\frac{(1-\theta)}{\theta}\left(Q_{i}^{n}-Q_{i+1}^{n}\right)
\end{aligned}
$$

y la ecuación de movimiento se reduce a:

$$
-Z_{i}^{n+1}+c Q_{i}^{n+1}+Z_{i+1}^{n+1}+d Q_{i+1}^{n+1}=e
$$

donde,

$$
\begin{gathered}
c=f-g_{1}+h \quad d=f+g_{1}+h \\
f=\frac{\Delta x_{i}}{2 \theta g \tilde{A} \Delta t} \quad g_{1}=\frac{2 \beta \tilde{Q}}{g \tilde{A}^{2}} \\
h=\frac{\chi \Delta x_{i} n^{2}|\tilde{Q}|}{2 \theta \tilde{A}^{2} \tilde{R}^{\frac{4}{3}}}
\end{gathered}
$$

$$
\begin{gathered}
e=\frac{(1-\theta)}{\theta}\left(Z_{i}^{n}-Z_{i+1}^{n}\right)+\left[f+\frac{(1-\theta)}{\theta} g_{1}-\frac{(1-\chi)}{\chi} h\right] Q_{i}^{n} \\
+\left[f-\frac{(1-\theta)}{\theta} g_{1}-\frac{(1-\chi)}{\chi} h\right] Q_{i+1}^{n} \\
+\frac{\beta \tilde{Q}^{2}}{\theta g \tilde{A}^{3}}\left[\tilde{T}\left(\tilde{Z}_{i+1}-\tilde{Z}_{i}\right)+\left.\left(\tilde{A}_{i+1}-\tilde{A}_{i}\right)\right|_{Z=c t e}\right]
\end{gathered}
$$

Las ecuaciones algebraicas lineales (10) y (12), que definen el flujo para el segmento $\Delta x_{i}$, se pueden expresar también en la siguiente forma matricial [5]:

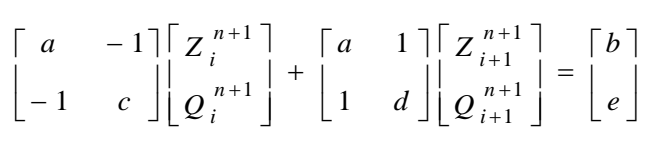

Entonces, se obtiene un par de ecuaciones algebraicas lineales por cada segmento de la grilla $(i, i+1)$;

$$
\begin{aligned}
& a_{i} Z_{i}^{n+1}-Q_{i}^{n+1}+a_{i} Z_{i+1}^{n+1}+Q_{i+1}^{n+1}=b_{i} \\
& -Z_{i}^{n+1}+c_{i} Q_{i}^{n+1}+Z_{i+1}^{n+1}+d_{i} Q_{i+1}^{n+1}=e_{i}
\end{aligned}
$$

Para ii puntos de cálculo se generará un sistema de (2ii-2) ecuaciones algebraicas lineales con $2 i i$ incógnitas. Por lo que se necesita adicionar dos ecuaciones, las que provienen de las condiciones de frontera, para completar el número de ecuaciones necesarias. Luego de lo cual el sistema podrá ser resuelto.

\subsection{Canal Ejemplo de Cuatro Segmentos}

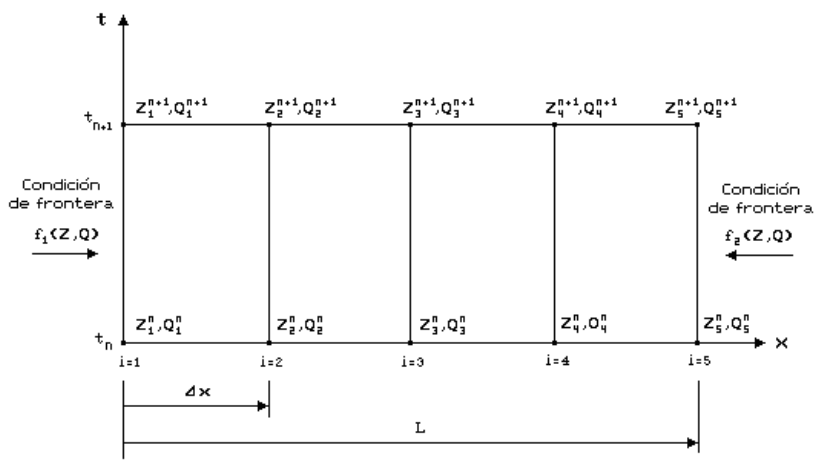

Fig. 3. Grilla de cálculo para un canal de 4 segmentos

Para un canal de 4 segmentos $(n d x=4)$ y 5 puntos de grilla $(i i=5)$, se obtiene el siguiente sistema de ecuaciones lineales, $A x=b$ : 
(16)

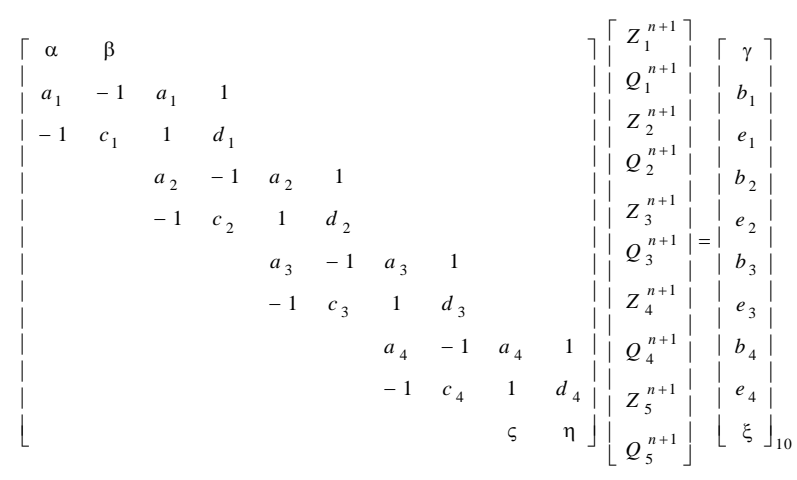

Es decir, se obtiene un sistema matricial con una matriz de coeficientes pentadiagonal, $A$, de orden $2 i i$, un vector de incógnitas, $x$, de tamaño $2 i i$, y un vector de residuos, $b$, de tamaño $2 i i$.

El sistema matricial puede resolverse, con las condiciones iniciales apropiadas, mediante el método de Eliminación de Gauss o también utilizando el método de Doble Barrido [8].

Después de resolver el sistema de ecuaciones se proporcionan los valores calculados a las variables del nivel de tiempo $(n+1) \Delta \mathrm{t}$ (la primera aproximación en el proceso de iteración fue al adoptar los valores del tiempo precedente). Luego, los coeficientes de las ecuaciones lineales pueden ser recalculados y actualizados y el sistema podrá ser resuelto de nuevo. Este proceso necesita de dos a tres iteraciones para obtener una muy buena aproximación, pero ya en la segunda iteración se obtiene una solución satisfactoria.

\subsection{Condiciones de Frontera Externas}

La solución de las ecuaciones de flujo requiere que se especifiquen condiciones de frontera en los extremos del canal durante todo el tiempo de simulación para proveer el número suficiente de ecuaciones adicionales y satisfacer los requerimientos de la técnica de solución.

Estas condiciones de frontera pueden ser elaboradas a partir de registros históricos o calcularse mediante funciones especificadas por el usuario. Se tienen varias combinaciones de condiciones de frontera externas, estas pueden consistir de una descarga cero (por ejemplo, al final del canal), un caudal o nivel conocido en función del tiempo, o una curva de calibración conocida. Las condiciones de fronteras tipo series de tiempo, pueden ser leídas por el programa desde archivos de datos [9].

También se ha implementado una condición de frontera no reflectante, la cual consiste de una relación matemática que permite que las perturbaciones u ondas pasen libremente por la frontera y no se reflejen y regresen dentro del dominio de cálculo [7].

A. Algoritmo general para condiciones de fronteras aguas arriba:

$$
\alpha Z_{1}+\beta Q_{1}=\gamma
$$

$$
\begin{aligned}
& \text { Si se proporciona } Z_{1}: \quad \alpha=1 \\
& \beta=0 \\
& \gamma=Z(t) \\
& \text { Si se proporciona } Q_{1}: \quad \alpha=0 \\
& \beta=1 \\
& \gamma=Q(t)
\end{aligned}
$$

B. Algoritmo general para condiciones de fronteras aguas abajo:

$$
\varsigma Z_{i i}+\eta Q_{i i}=\xi
$$

$$
\text { Si se proporciona } Z_{i i}: \quad \begin{array}{ll}
\varsigma & =1 \\
& \eta=0 \\
& \xi=Z(t) \\
\text { Si se proporciona } Q_{i i}: & \varsigma=0 \\
& \eta=1 \\
& \xi=Q(t)
\end{array}
$$

\subsection{Condiciones Iniciales}

Para iniciar la solución del sistema de ecuaciones algebraicas lineales, se requieren los valores de las variables de flujo para el tiempo cero. Tales valores se pueden obtener de datos medidos o calculados de alguna otra fuente, tales como aproximaciones para un estado permanente, o resultados de alguna simulación anterior.

El uso sucesivo de los valores calculados como las nuevas condiciones iniciales permite que el proceso de cómputo proceda paso a paso hasta concluir la simulación. Una convergencia exitosa del cómputo a la solución correcta requiere que los valores iniciales sean 
razonablemente precisos; a menor precisión de los valores iniciales, mayor tiempo se tomará para disipar los errores iniciales y llegar a la solución correcta.

\subsection{Procedimiento de Solución}

El procedimiento de simulación se realiza para cada paso de tiempo; es decir, dadas las condiciones iniciales en el tiempo $t_{0}$, donde todos los valores de las variables se conocen $\left(Z_{i}^{n}, Q_{i}^{n}\right)$, procedemos al cálculo del paso de tiempo siguiente, $t_{1}$, en el cual, dada una condición de frontera aguas arriba; por ejemplo, un aumento de caudal (el cual debe propagarse hacia aguas abajo) procedemos al ensamblaje de las ecuaciones correspondientes en todos los puntos de grilla a lo largo del tramo en estudio. Luego, adicionamos la ecuación correspondiente a la condición de frontera aguas abajo (que puede ser una función de vertedero por ejemplo). Así, completamos el sistema de ecuaciones que procederemos a resolver mediante el método de Doble Barrido [9].

La solución del sistema de ecuaciones proporciona los niveles y caudales en todos los puntos de la grilla $\left(z_{i}^{n+1}, Q_{i}^{n+1}\right)$. Se pueden realizar iteraciones dentro de un mismo paso de tiempo para mejorar la precisión de la solución. El efecto primario de la iteración es mejorar las cantidades tomadas como constantes locales en el paso de tiempo, los cuales a su vez incrementan la precisión de las variables calculadas. Una buena precisión es normalmente alcanzada con dos o tres iteraciones por paso de tiempo.

Seguidamente se almacena la solución obtenida y se transfieren dichos valores al vector que contiene las condiciones iniciales o variables del tiempo $t_{0}$.

Para el segundo paso de tiempo se procede de manera idéntica al proceso anterior. Este procedimiento se repite hasta completar todos los pasos de tiempo que requiere la simulación. Por ejemplo, se puede tratar de una onda que toma dos horas en pasar por el extremo aguas arriba del canal, entonces, la simulación frecuentemente debe tomar en consideración además el tiempo que la onda demora en llegar al punto de interés o en pasar totalmente por el extremo aguas abajo del canal. Por todo esto, el tiempo total de simulación en casos reales es frecuentemente grande [9].

\section{Pruebas Esquemáticas}

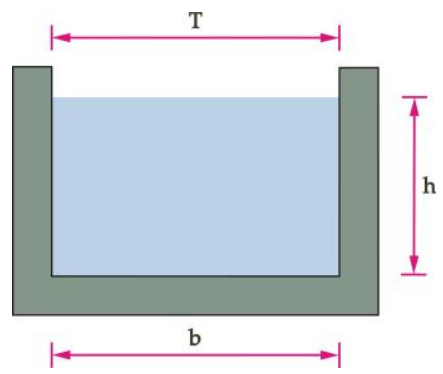

Estas pruebas consisten en simular flujo estacionario y no estacionario en un canal singular. El canal tiene una longitud total de 10 kms y una pendiente de fondo $\left(S_{0}\right)$ igual a $1 \times 10^{-5}$. La sección transversal es rectangular prismática y tiene un ancho de $100 \mathrm{~m}$. Cada segmento tiene una longitud de $1000 \mathrm{~m}$ $(\Delta x=1000 \mathrm{~m})$, por lo que se tienen 10 segmentos $(n d x=10)$ y 11 secciones transversales $(i i=11)$.

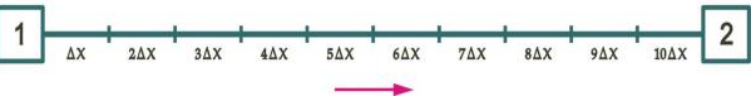

El tiempo total de simulación es de 12 horas, con un paso de tiempo $(\Delta t)$ de $600 \mathrm{seg}$., lo que produce 72 pasos de tiempo. El coeficiente de rugosidad de Manning ( $n$ ) es constante e igual a 0.026 en todo el sistema, $\beta=1.0 \mathrm{yg}=9.81 \mathrm{~m} / \mathrm{s}^{2}$.

\subsection{Test de Flujo Estacionario}

Los factores de ponderación del esquema Preissmann tienen los siguientes valores $\theta=0.7, \psi=0.5, \chi=0.5$. Las condiciones iniciales y de frontera para el canal se resumen en el siguiente cuadro:

\begin{tabular}{|c|c|c|}
\hline \multicolumn{3}{|c|}{ CONDICIONES INICIALES } \\
\hline $\begin{array}{c}\text { Caudal } \\
\text { Qo }\left(\mathrm{m}^{3} / \mathrm{s}\right)\end{array}$ & $\begin{array}{c}\text { Altura } \\
\text { ho }(\mathrm{m})\end{array}$ & $\begin{array}{c}\text { Velocidad } \\
\text { Vo }(\mathrm{m} / \mathrm{s})\end{array}$ \\
\hline 500 & 10 & 0.50 \\
\hline
\end{tabular}




\begin{tabular}{|l|l|}
\hline \multicolumn{2}{|c|}{ CONDICIONES DE FRONTERA } \\
\hline Frontera Aguas Arriba & $\mathrm{Q}=500 \mathrm{~m}^{3} / \mathrm{s}$ \\
\hline Frontera Aguas Abajo & $\mathrm{Q}=500 \mathrm{~m}^{3} / \mathrm{s}$ \\
\hline
\end{tabular}

Los resultados se han registrado durante las 12 horas de simulación en 5 estaciones de control ubicadas en $0,2,4,6,8$ y $10 \mathrm{kms}$. Los hidrogramas muestran que los niveles y los caudales permanecen constantes durante todo el tiempo de simulación. Excepto en la primera media hora, que se presentan algunas fluctuaciones de caudales; pero que no exceden de $0.07 \mathrm{~m}^{3} / \mathrm{s}$. Los perfiles longitudinales registrados a las $0,3,6,9$ y 12 horas muestran un perfil constante a lo largo de toda la longitud del canal.
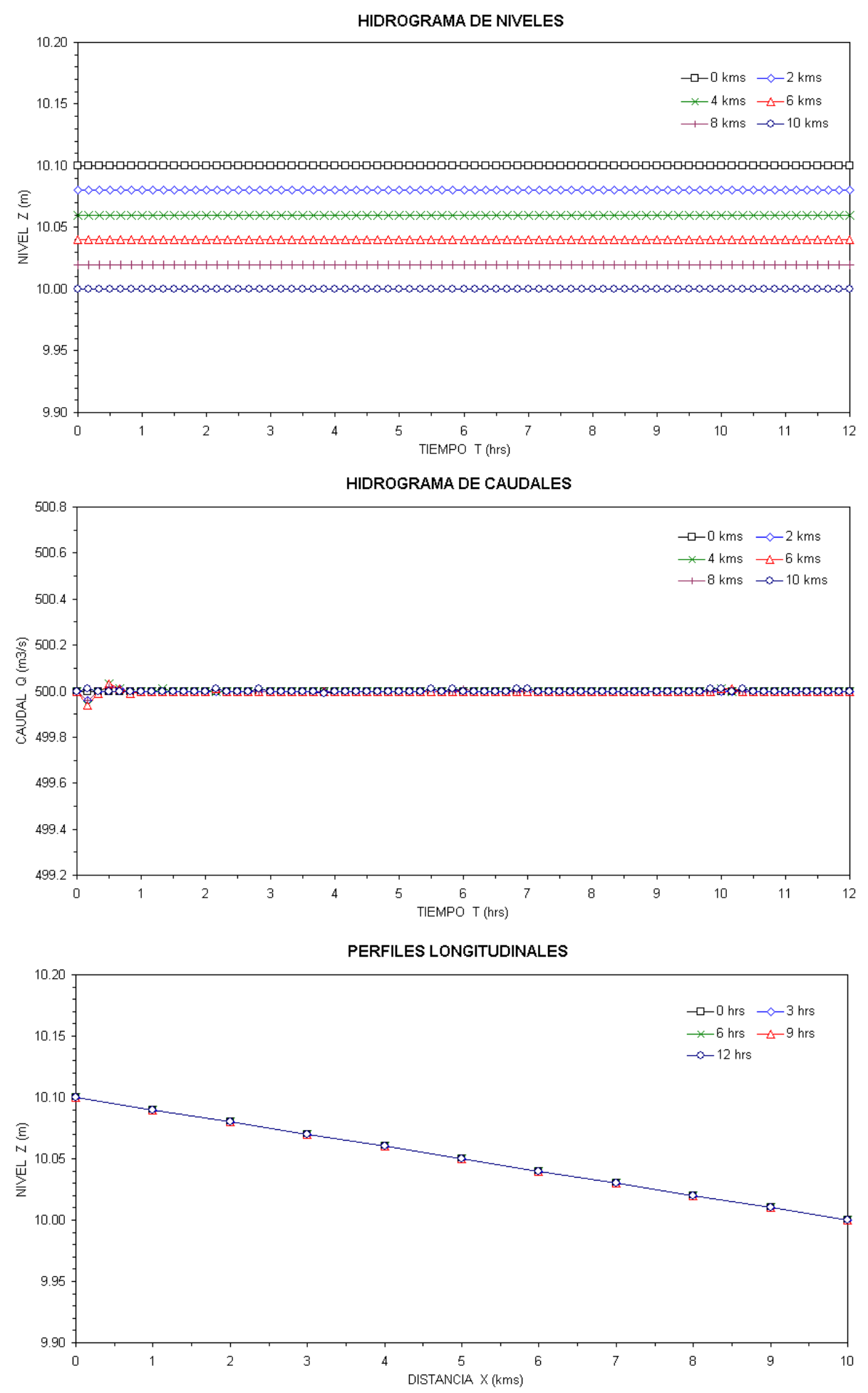


\subsection{Test de Flujo No Estacionario}

Los factores de ponderación del Esquema Preissmann tienen los siguientes valores $\theta=0.8, \psi=0.5, \chi=0.5$. Las condiciones iniciales y de frontera para el canal se resumen en el siguiente cuadro:

\begin{tabular}{|c|c|c|}
\hline \multicolumn{3}{|c|}{ CONDICIONES INICIALES } \\
\hline $\begin{array}{c}\text { Caudal } \\
\text { Qo }\left(\mathrm{m}^{3} / \mathrm{s}\right)\end{array}$ & $\begin{array}{c}\text { Altura } \\
\text { ho }(\mathrm{m})\end{array}$ & $\begin{array}{c}\text { Velocidad } \\
\text { Vo }(\mathrm{m} / \mathrm{s})\end{array}$ \\
\hline 500 & 10 & 0.50 \\
\hline
\end{tabular}

\begin{tabular}{|c|c|}
\hline \multicolumn{2}{|c|}{ CONDICIONES DE FRONTERA } \\
\hline Frontera Aguas Arriba & Hidrograma (Q) \\
\hline Frontera Aguas Abajo & Radiación (Q) \\
\hline
\end{tabular}

La condición de frontera aguas arriba es un hidrograma de caudales del tipo Log-Pearson III, y la condición de frontera aguas abajo es del tipo no reflectante, desarrollada a partir de la ecuación de radiación unidimensional.

Los resultados de la prueba se han registrado durante las 12 horas de simulación en las 5 estaciones de control ubicadas en 0, 2, 4, 6, 8 y $10 \mathrm{kms}$.

Los hidrogramas resultantes muestran que la variación de niveles y de caudales reproducen aceptablemente el flujo no estacionario. Los perfiles longitudinales registrados a las $0,2,4,6$ y 12 horas muestran una variación que concuerda con los registros del hidrograma de niveles en todas las estaciones de control.
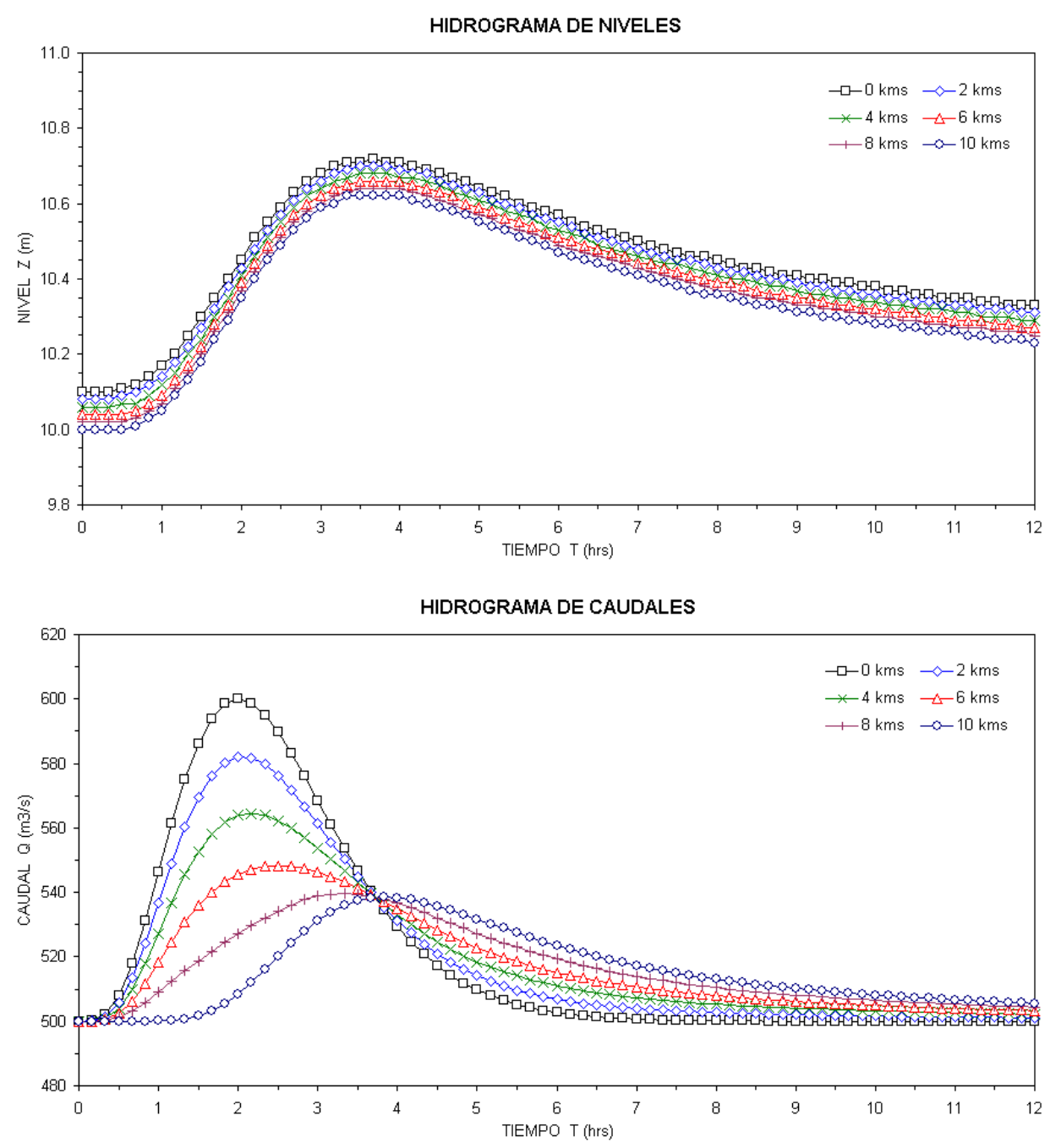


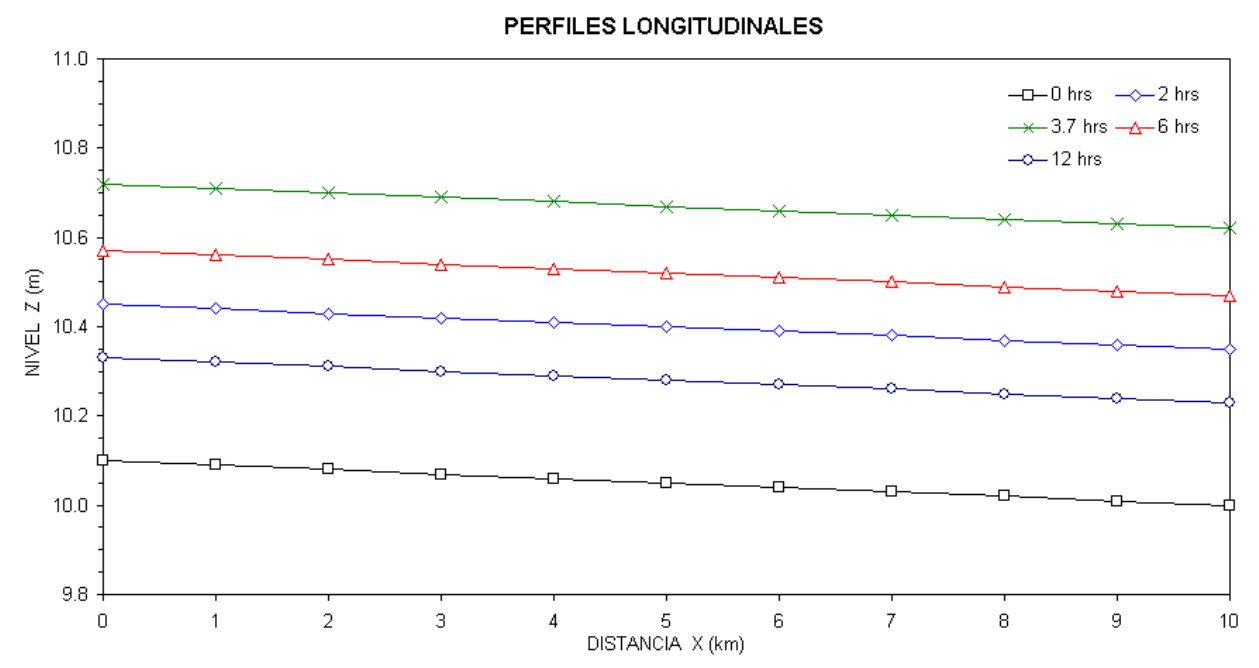

\section{Conclusiones}

El trabajo presentado demuestra que el modelo produce buenos resultados en la simulación del flujo unidimensional en canales singulares. Los resultados de las aplicaciones ilustran la flexibilidad y precisión del modelo para simular diversas condiciones de flujo, tales como flujo estacionario uniforme y no uniforme, y también flujo no estacionario en canales abiertos artificiales.

La técnica de diferencias finitas implícito de Preissmann con sus coeficientes de ponderación confiere un alto grado de flexibilidad para simular diversas condiciones de flujo en canales de propiedades constantes o variables. Además, permite utilizar grandes pasos de tiempo y también discretizar el canal con segmentos de longitudes desiguales.

El modelo también cuenta con el coeficiente de Boussinesq, el cual permite tomar en cuenta las distribuciones de flujo no uniforme, que se presentan en las secciones con cambios bruscos de geometría y también en los tramos con curvas.

Las pruebas mostradas permiten asegurar que es posible utilizar el modelo para realizar el diseño de canales, modificando la geometría del mismo para obtener la curva de remanso deseada, sin cambios abruptos y evitar grandes pérdidas de carga.

También debe resaltarse que el modelo es hidrodinámico y no contempla el transporte de sedimentos ni los cambios morfológicos del cauce.

\section{Referencias}

[1] Abbott M. B., "Computational Hydraulics", Pitman Publishing Limited, London, 1985. $326 \mathrm{pp}$.

[2] Abbott M. B., Basco D. R., "Computational Fluid Dynamics, An introduction for Engineers", Longman Scientific \& Technical, London, 1989. 425 pp.

[3] Cunge J. A., Holly F. M. and Verwey A., "Practical Aspects of Computational River Hydraulics", Pitman Publishing Limited, London, 1980. $420 \mathrm{pp}$.

[4] Chow V. T., "Open Channel Flow", McGraw Hill Book Company Inc. New York, 1964.

[5] Schaffranek R. W., "Flow Model for Open Channel Reach or Network", U.S. Geological Survey, Professional paper 1384, 1987.

[6] Strelkoff Theodor, "One-dimensional Equations of Open Channel Flow", ASCE, Journal of the Hydraulics Division, v. 95, $\mathrm{N}^{\circ}$ HY3, p. 861-876, 1969.

[7] Vreugdenhil, C. B. Computational Hydraulics. Springer Verlag, Berlin, 1989. $182 \mathrm{pp}$.

[8] Yzocupe V. A. "Simulación del Tránsito de Avenidas (Flood Routing)", Tesis profesional. UNMSM, EAP de Ingeniería Mecánica de Fluidos. Lima, 1993. 220 pp.

[9] Yzocupe V. A. "Modelamiento Numérico en Ingeniería Hidráulica". Laboratorio de Fluidodinámica Computacional - UNMSM. Lima, 2004. 110 pp. 